\title{
Design Educator Education - Creating Effective Judging and Teaching Teams in Engineering Design Contests and Courses
}

\author{
Patricia K. Sheridan \\ PhD Candidate \\ University of Toronto \\ patricia.sheridan@utoronto.ca
}

\author{
Alan Chong \\ Senior Lecturer, \\ Engineering Communication Program \\ University of Toronto \\ alan.chong@utoronto.ca
}

\author{
Jason A. Foster \\ Senior Lecturer, \\ Div. of Engineering Science \\ University of Toronto \\ jason.foster@utoronto.ca
}

\begin{abstract}
In this paper we employ Tuckman's model of team development to understand how judging and teaching teams form in the context of design contests and courses. A poorly developed team can negatively influence student or participant experience at these events. Tuckman's Form-Storm-Norm-Perform model provides insights into how such teams can fail to develop and, we argue, suggests strategies and approaches for improved team orientations, meetings, and rubric development practices that help develop more effective judging and teaching teams.
\end{abstract}

\section{Introduction}

Engineering design education brings together participants with diverse experiences and perspectives on engineering design, including faculty members, practicing engineers, university administrators, and graduate, undergraduate, and high school students playing a variety of roles as coordinators, judges, and teachers to name a few. For the courses and contests in which engineering design is practiced, this creates a challenging team dynamic: each participant, based on experience and education, brings their own perspective on the nature, practice, and assessment of engineering design to the team. Some may privilege innovative conceptual design, others evidence of specific processes or tools, while some focus solely on cost, safety, and functionality. In addition to their perspectives on engineering design, the participants also bring their own understanding of the goals of the various activities. University administrators may focus on the potential for outreach and image enhancement, whereas practicing engineers may emphasize attracting new members to the engineering profession. Additionally, the engineering design courses and contests themselves also embody specific goals and perspectives on both engineering practice and the roles of the participants.
Such diverse views are both a boon and bane to engineering design pedagogy and practice. These differences allow for a plurality of perspectives, but a successful engineering design course or contest requires at a minimum harmony among the perspectives of the participants, and between these perspectives and those of the design experience. In the absence of such harmony, student or participant satisfaction and engagement may be compromised as they receive mixed or contradictory guidance and assessment. Based on past experience in both courses and contests, this synthesis of perspective is not always readily attainable, especially within tight time constraints. The high turnover, independent culture, and lack of incentives within the academic environment makes it exceedingly challenging to form effective judging and teaching teams.

One solution to these challenges involves applying the formal literature on team formation often taught to students in design courses to the practice of constructing design experiences. In particular, this paper shows how strategies borne from Tuckman's model of team development [1] - a canonical model can be used to illustrate specific challenges in team taught engineering design courses and team organized and judged engineering contests, and to provide strategies for addressing these challenges. In this paper, we will present some of the issues that occur in the formation of teaching and assessment teams in two design experiences at the University of Toronto: Praxis, and the University of Toronto Engineering Kompetitions (UTEK). Specifically, we identify challenges in the rapid development of shared objectives, values, and assessment criteria for engineering design through the lens of Tuckman's model of team development. Subsequently, we discuss several strategies implemented in both design contexts to mitigate such issues. 


\section{Teams in Design Experiences}

Before we begin, however, we do need to acknowledge that courses and contests themselves present different engineering design situations, with different goals and challenges, and different ways of deploying team members. In large scale engineering design courses, which take place over the course of a term or a year, several faculty members may be instructors or coordinators, but on the ground, these courses are often staffed with industry experts as adjunct instructors, project managers, or even as clients. Academic proto-experts, such as graduate students and advanced undergraduates, may also serve as teaching assistants. In such an environment, a shared understanding of the course vision or learning objectives, as outlined in the syllabus, is essential, though there is time to develop such a shared vision. In a contest situation, however, time is extremely limited. In a contest, faculty members may act both as mentors to the competition organizers and as judges of the participants' designs. In both capacities, an accurate understanding of the criteria for the design assessment is vital, to both ensure that the competition reflects the stated goals and vision, and to ensure students are fairly judged and appropriately guided.

While generalizable to other contexts, the issues identified in the formation of assessment and teaching teams do come primarily from two highly specific design experiences, which we first describe.

Praxis is a two term cornerstone design course for first year Engineering Science students that is a personal growth design experience. The nature of this course is to have each student develop and credibly practice design along their particular philosophy of engineering design. Introduced to a variety of models and approaches to design, students develop their own definitions of what engineering design is, and what it means to be both an engineer and a designer. The design activity in this experience is that of an instructor-guided, but student-developed project, where students must determine both the design problem and solution that remedies an environmental defect provided by the instructor. This course of approximately 250 students is taught by a 10 person teaching team, where the vision of the course must be messaged in the teaching and assessment practices of all members of the team. The individual beliefs of the teaching team in engineering design must not be messaged to the students, as this would impede the development of the students' individual understanding and perspectives on engineering design.

University of Toronto Engineering Kompetitions (UTEK), is a faculty-wide design and communication competition to determine the best competitors to represent UofT at the Ontario, and possibly Canadian, Engineering Competitions. In the context of this paper, we will examine solely the judging teams of the design competitions (Junior, Senior, and Innovative Design), wherein teams of students are asked to provide a solution to either a given problem (Junior/Senior) or a problem they determine (Innovative). Judging teams of 3-5 members per competition are formed on the day of the contest, and require judges to develop a synthesized vision of the objectives of the competition, and criteria used to assess the teams relative performances rapidly. The individual perspectives of the judges on the importance of different aspects of engineering design must be aligned with those of the organizers to develop a consistent set of judging criteria to objectively assess the participants.

\section{Common Team Development Issues in these Design Experiences}

Despite the major differences in the contexts cited above, both have suffered from similar challenges arising from team development issues, particularly in the development of shared values and in how those values translate into assessment.

\subsection{Issues in the Synthesis of the Organizers and Assessors Values}

In classes where the subject matter is open to interpretation, such as Praxis, a synthesis in the values of the teaching team is necessary to facilitate a consistent messaging of, and assessment of the students according to, the core values of the course. In a design course, the course syllabus or stated learning objectives, formulated by the instructor or coordinator, forms the basis of the normative values that are developed through the class. These values are often articulated through lectures, assignments, and in instructor meetings over the course of the term. In more rare cases, they can be disseminated through teaching assistant training sessions; more often than not, however, these training sessions focus on consistency in the content and delivery of the assignments, rather than the development of shared values and learning outcomes. Shared approaches and practices are not equivalent to shared values, and can result in vastly different experiences across classrooms. Without this synthesis, each member of the teaching team presents to their section their interpretation of the values, privileging different values based on education and experience, resulting in 
an inconsistency in the objectives and assessment methods presented across the course.

Our experience shows that these issues often take a while to surface; there is often a perceived but superficial agreement on key values that only begins to break down as we begin to explore more subtle elements of the course. These discontinuities are usually communicated through teaching team meetings, or inconsistencies present in students' understanding of the course objectives. Once these issues have been flagged, the teaching team then needs to identify the inconsistencies between their values, and develop a consistent value set for the course.

In design contests such as UTEK on the other hand, there are fundamentally more issues in the privileging of different values across assessors that are not always mitigated as a result of time constraints on team development. Brief orientation sessions, facilitated by one of the contest organizers, are the only place in which assessors can identify and unify their values with each other's, and with the organizer's. These sessions, of no more than an hour in length, introduce the judges to each other, outline the components of the contest, and walk the judges through the assessment forms (criteria). These assessment forms and any values messaged during the briefing form the basis of the normative values that the organizers want the assessors to privilege. Organizers often fail to define the evaluation criteria, however, as they feel that their definitions of the criteria are intuitive, and do not explore the many possible interpretations privileged by the assessors to find a common one. These issues are further compounded by the organizers not clearly identifying what their objectives are in terms of the experiences of the participants. However, since no feedback is provided to the participants of UTEK postcontest other than an ordinal ranking, the need to develop this synthesis in values, assessment criteria and their relative importance during the briefing is not always apparent.

These issues, similarly take a while to surface, and are often not readily apparent until the judges meet at the end of assessment to determine the winners. At this point the judges realize, that without acknowledging it, they assessed the participants with emphasis on different values. This is largely due to the fact that the assessors share a multitude of backgrounds, which frame their objectives of the experience for the participants differently than those of the organizers. In particular, the most common issue is the relative importance of the team's conceptual design in comparison to their ability to construct a functioning prototype. This leaves the adjudication sessions post-assessment as the place where determination of the key values of the contest occur, creating difficulty in assessing all teams according to the same values and criteria.

\subsection{Issues with Assessment Rubrics}

One key strategy to enforce consistency in visioning, at least in the messaging through assessment, involves the use of rubrics. However, rubric development generates another set of problems for course and contest organizers. Generic or simple rubrics are of little value in defining assessment criteria, because the purposefully vague language allows significant subjectivity in interpretation. When these rubrics are overly complicated or simplistic, assessors tend to become hostile towards using them, and either ignore them, or use them inappropriately or superficially. These issues are apparent in both design courses and contest and can be attributed to the tractability of the assessment criteria used in them.

In design courses, if a shared vocabulary or set of values has been established, more detailed rubrics can be developed. In Praxis, such rubrics employ precise language to help establish criteria and levels of performance more clearly. For example, instead of rating a specific criteria along a scale, the assessor can select the level of performance in terms of a description of a level of inclusion of the criteria. For the students, these are particularly valuable, since they break down their performance into precise categories and levels. For actual assessment, however, these rubrics are challenging to use: they take significant amounts of time to complete; even without the holistic comments, having to choose between four levels of performance in 30 categories, especially in the 15 minutes spent listening to a presentation or viewing a demo, can be a real challenge. Moreover, though the checkboxes are well defined, actual numerical grades assigned to the various levels of performance tend to vary between instructors. Assessment rubrics imply an agreement on what constitutes a certain level of performance in a specific category, as well as on numerical values associated with these levels of performance; however, such agreement is often not achieved resulting in a variety of uses of the rubrics.

In design contests, the value of the assessment rubrics is often diminished by the lack of definition of the criteria utilized, as well as the lack of relevance of the criteria to developing an assessment of participants' abilities. In many cases, the inability to determine how to assess the criteria, and the lack of definition of what they mean discount from the credibility of the rubrics. For example, it is difficult to assess the sustainability of a design on a scale from 1 to 5 without a definition of what aspects of 
sustainability are being assessed, and what metric is being used. However, given the time scale of the contest (one day) developing such well-defined rubrics as is done in design courses is equally infeasible, as the assessors would shun the complexity of evaluation. At UTEK, since judges are only requested to provide an ordinal ranking of the students, judges have tended to disregard the actual rubrics, and attempted to evaluate the participants somewhat according to the suggested criteria. At this point, assessors may, consciously or subconsciously, introduce their own assessment criteria such as the visibility of women or minorities on the winning teams, or the use of specific design tools or processes that they are familiar with, or fond of. This ends up resulting in a subjective assessment of the teams, which privileges not only different assessment criteria but different understandings of these criteria.

\section{Tuckman's Model of Team Development}

Tuckman's model of team development breaks the process into four distinct stages: forming, storming, norming, and performing [1]. It is often introduced in team based engineering design courses in order to provide students with an idealized process which can help them to understand and improve their team experience, as well as achieve their goals more rapidly. In the first stage, Forming, the team is introduced to each other, is caught up primarily in information gathering and team organization, with very little conflict and issues of substance being discussed. The Storming stage follows, in which team members begin to put forward their own competing ideas about team organization, goals, and vision. In this stage, the individual is prominent, with a strong resistance to group influence. Although it seems contrary to team functioning, the Storming stage is an essential precursor to Norming, in which Storming conflicts are resolved, and a shared team vision is created. With a shared vision, teams can move onto Performing, addressing the task at hand as a cohesive team. Of course, Tuckman's model is idealized: teams are often left at the storming stage, and left unable to perform. Sometimes, teams might experience a "False Norming," in which superficial agreement is reached, with deeper divisions never having been articulated or expressed. These issues prevent appropriate Performing, or result in a return to Storming later on in the process.

\section{Issues as seen through the Tuckman Model}

Team development is a necessity in both design courses and contests, but under radically different conditions. The different time constraints and need for transparency in assessment greatly vary the way in which teams can develop a synthesized vision of the experience, and participant assessment. Ultimately, the amount of time the team can spend working through the Tuckman model, and training strategies to facilitate this, affects the ability of the team to develop this synthesis.

However, based on experience in these design contests and courses at UofT, two key issues arise in the development of these assessment teams. The first issue is the undervaluing of the Storming phase. This stems from assessors and organizers believing that the other team members hold the same definitions and values of design that they do, and will privilege the weighting of assessment criteria as they do. As a result, the value in discussing different interpretations is not seen by the assessors. The second issue is a sense of false norming created by joint assessment tools, such as rubrics, that leave too much room for interpretation. Here, assessors experience a belief of unity in what the evaluation criteria are and how to assess them, without the assessors storming to develop a consistent set of criteria, and their relative importance, with which to assess. To develop a team with a synthesized vision, strategies to develop these must focus on demonstrating the importance of Storming to assessors, and the ability for overly broad rubrics to create false norming.

\section{Strategies to Avoid Team Development Issues}

\subsection{Design Course - Strategies in Praxis}

To address differences in values across the teaching team, training in Praxis has the teaching team engage in the same exploration of personal engineering design values that the students taking the course experience. Simultaneously novice instructors are exposed to the Praxis teaching values through direct exposure. This exploration and exposure is accomplished by focusing on the student experience of course activities, rather than on the mechanics of the activities the instructors be facilitating. In these training activities, performing as a member of the teaching team or a student in a tutorial is simulated such that storming on the issues relevant to effectively teaching these classes can be identified. For example, instead of explaining how to run the first course activity, seasoned instructors run new instructors through the activity. This allows the new instructors to identify with the experience from the perspective of the students. For example they experience the value placed on the students' own 
opinions and understanding of engineering design, and helps to set the tone for the course. The new instructors are also prompted to "freeze" of the activity when their instinctive approach to facilitation differs from that which they have experienced. Such freezing provides a space within the simulation that allows the teaching team to develop common norms based on their direct experience as facilitators and simulated students.

An enhancement to the approach just mentioned is based on an activity borrowed from improvisational theatre. While simulating the activity, the participants can call for a "repeat" or a "switch". Repeating prompts those within the simulation to try a new approach to their most recent interaction; switching allows a participant to take on a different role and attempt a different approach to the situation. In both cases the teaching team as a whole is exposed to different ways of reacting to situations that they will encounter in the classroom. This activity becomes extremely valuable when a veteran instructor "switch"es into the role of a student in order to model a negative behaviour that they have had to address. Given that one of the core goals of Praxis is to promote self-directed exploration by the students, this activity allows the new instructors to identify the types of questions and answers that provide solutions to, or promote students in further inquiry to answer their own questions. This re-iterates the objectives of the course and the trends and implications of instructorperspective messaging so that they can notice and avoid such teaching habits.

The result of these simulated activities is that the members of the teaching team explore their own values as engineering designers, experience the values of other engineers, and start to incorporate teaching practices that have been demonstrated to be successful into their approach to design education.

As stated previously, appropriate rubric development is essential to good norming on assessment. Rubrics with a large number of categories and levels of performance are time consuming to produce and difficult to use, but valuable for students. Based on feedback from our assessors, In Praxis, we have begun to develop two versions of the rubric, one for use during assessment, and one - more detailed - to be delivered to the students and completed after the assessment session. These two tiers of rubrics provide the assessors with sufficient information to assess according to the pre-normed values, but not so much that they are overwhelmed and distracted by the rubric while assessing.

When possible, we involve the teaching team in the initial development of our rubrics through a guided development process. Iterating between individual and group development phases, the teaching team first develops the rubric criteria. For each criteria, we then identify key features that we anticipate will distinguish between different levels of performance. The course instructors finish by synthesizing a complete rubric.

Regardless of when or how a rubric was developed, a calibration process is always followed when the rubric is used for assessment. When resources permit, all assessors meet and grade a common set of assignments. Holding this meeting face to face allows the teaching team to immediately identify and resolve any differences of opinion or interpretation. These resolutions are immediately incorporated into a revised rubric. If resources do not permit, then each assessor is asked to submit their initial feedback to the course instructors so that a common understanding can be reached using the instructors as intermediaries. This alternate approach can also result in modified rubrics. In essence, the rubrics follow the same iterative storm, norm, and perform cycle to ensure they convey the intended meaning and are not confusing to use.

Finally, team meetings need to be deeply informed by an awareness of the non-linearity of Tuckman's model. More importantly, regular team meetings (as many as twice per week) are essential for revisiting the team development process. Armed with an awareness of the high possibility for "False Norming," assumptions about agreement are constantly revisited and the discussion of values, classroom practices, and their intended objectives are encouraged. As a result, more time is spent storming in these meetings than norming. A typical weekly cycle of team meetings begins with an ad hoc meeting after the final lecture but prior to the studios. At this meeting any anticipated issues arising from the lecture materials are raised. After the first section of each week has completed running its studio, the teaching team meets to reassess the objectives of the activity, how it was received by the students, and its value to conveying the vision of the course. Each member is prompted to provide feedback about what did and did not work well, with confrontation not considered a barrier to progress. The course instructors model an open, occasionally confrontational approach to these meetings to elicit as much and as authentic feedback and discussion as possible.

Following this second meeting, the studio activities may be slightly, or even completely reworked until the team finds a structure that meshes with both their values and approach to teaching, and that of the course. This constant re-storming and re-norming of the team's visions and methods of executing them 
ensures that during performing the students receive the intended messaging from the activities.

\subsection{Design Contest - Strategies at UTEK}

The strategies used to address team development issues are different between contests and courses due to the vast differences in time scale available to develop team synthesis. At UTEK, judges are briefed the morning of the contest, and then spend the day assessing the designs of the participants. This briefing is the main place where the judging team development occurs, and as a result needs to move the judges through the forming, storming and norming stages in less than an hour, so that they can perform for the remainder of the day. While the forming stage is intuitive to the judges, strategies are needed to push them to the storming and norming stages during this briefing.

Many of the strategies we have used at UTEK have focused on developing an agreed upon and workable set of assessment criteria that meet the goals of the organizers. We have focused on these areas rather than improving the training of the assessors or the level of detail of the rubrics simply due to the time constraints and the need for a fast norming. The focus of these strategies is to force storming activities between the assessors and contest organizers with respect to the assessment criteria before any participants are assessed. This way a normed vision of what defines a winning team can be determined pre-assessment, and all participants assessed objectively. The strategies discussed herein are a combination of those which were incorporated into the contest previously, and those to be incorporated based on lessons learned at the previous contest.

One strategy we have used in the past to accelerate the judging teams towards the storming stage is to include at least one design educator and/or one past judge on each judging team. The inclusion of a design educator promotes broader perspectives on design, as they can challenge the stereotypical definitions of design that many industry judges may privilege. Additionally, both members are able to identify and promote the discussion of criteria based interpretation issues in the rubrics as they recognize the criteria which commonly have the greatest amount of interpretation. In the past few years, this has promoted the determination of at a minimum the most important aspects of the design to assess, and a vision for how these aspects manifest in the potential design solutions. As a result, regardless of whether the provided rubrics were utilized or acted solely as a catalyst for discussion, a unified, or normed, vision of the assessment criteria and winners was determined.

An additional strategy to facilitate the storming and norming discussions is to provide the assessors with the questions participants asked during design development. By presenting them with the problems the participants had, and the responses provided by the organizers, helps the assessors determine where the ambiguities exist, as well as the values of the organizers in this respect. This facilitates a deeper understanding of any ambiguities in the competition problem statement, which may be transferred into the judges' assessment criteria.

However, not only reactive but proactive steps need to be taken to identify ambiguities in the assessment criteria of the rubrics. Organizers of UTEK, at these briefings now share their vision and interpretation of the criteria and metrics, and prompt assessors to outline any ambiguous terms to ensure they understand what the organizer's want them to assess and how. To facilitate identification of these issues, some judges recommend that providing assessors with a sample design team and solution to assess according to the rubric/criteria presented would aid in promoting storming. By running through this process, it would help the assessors identify the less obvious criteria that have different meanings across the assessors. This strategy has not yet been tested, but holds promise based on precedence from its use in other courses.

\section{Conclusions and Future Work}

Team development is an essential aspect of producing effective engineering design experiences for students. Taking advantage of theories of team development already taught in design courses - essentially practicing what we preach - and adjusting our strategies for arriving at "normed" values and assessment criteria allows us to understand and address the key strategies for improvement. Of course, Tuckman's model and the strategies borne from it provide just a starting point for our own introspection: a deeper understanding of our own unique teams, however it is developed, will help inform and develop a better experience for all participants.

\section{References}

[1] B.W. Tuckman, "Developmental sequence in small groups", Psychological Bulletin, 63(6), 1965, pp. 384-399. 